\title{
Tension Gauge Tether Probes for Quantifying Growth Factor Mediated Integrin Mechanics and Adhesion
}

\author{
Tejeshwar C. Rao ${ }^{1}$, Alexa L. Mattheyses ${ }^{1}$ \\ ${ }^{1}$ Department of Cell, Developmental, and Integrative Biology, University of Alabama at Birmingham
}

\section{Corresponding Author}

Alexa L. Mattheyses

mattheyses@uab.edu

\section{Citation}

Rao, T.C., Mattheyses, A.L. Tension Gauge Tether Probes for Quantifying Growth Factor Mediated Integrin Mechanics and Adhesion. J. Vis. Exp. (180), e63529, doi:10.3791/63529 (2022).

\section{Date Published}

February 11, 2022

DOI

$10.3791 / 63529$

URL

jove.com/video/63529

\section{Abstract}

Multicellular organisms rely on interactions between membrane receptors and cognate ligands in the surrounding extracellular matrix (ECM) to orchestrate multiple functions, including adhesion, proliferation, migration, and differentiation. Mechanical forces can be transmitted from the cell via the adhesion receptor integrin to ligands in the ECM. The amount and spatial organization of these cell-generated forces can be modulated by growth factor receptors, including epidermal growth factor receptor (EGFR). The tools currently available to quantify crosstalk-mediated changes in cell mechanics and relate them to focal adhesions, cellular morphology, and signaling are limited. DNAbased molecular force sensors known as tension gauge tethers (TGTs) have been employed to quantify these changes. TGT probes are unique in their ability to both modulate the underlying force threshold and report piconewton scale receptor forces across the entire adherent cell surface at diffraction-limited spatial resolution. The TGT probes used here rely on the irreversible dissociation of a DNA duplex by receptorligand forces that generate a fluorescent signal. This allows quantification of the cumulative integrin tension (force history) of the cell. This article describes a protocol employing TGTs to study the impact of EGFR on integrin mechanics and adhesion formation. The assembly of the TGT mechanical sensing platform is systematically detailed and the procedure to image forces, focal adhesions, and cell spreading is outlined. Overall, the ability to modulate the underlying force threshold of the probe, the adhesion ligand, and the type and concentration of growth factor employed for stimulation make this a robust platform for studying the interplay of diverse membrane receptors in regulating integrin-mediated forces. 


\section{Introduction}

Cells have the intrinsic ability to sense, generate, and respond to mechanical forces, leading to changes in cellular phenotype and remodeling of the local microenvironment ${ }^{1,2}$. Forces play a crucial role in regulating many aspects of cell behavior, including adhesion, migration, proliferation, differentiation, and wound healing ${ }^{3,4}$. Aberrations in the bi-directional mechanical exchange between a cell and the microenvironment can lead to diseased states, including cancer $^{5}$. Numerous membrane receptors are involved in maintaining cell-matrix homeostasis; of these, integrins and epidermal growth factor receptor (EGFR) have robust synergy ${ }^{6,7}$. Classically, integrins establish the mechanical link between the microenvironment and intracellular cytoskeleton while EGFR regulates cell growth, proliferation, and survival ${ }^{8,9}$. EGFR is a highly studied therapeutic target, focused on outside-in regulation facilitating intracellular signaling. EGFR-integrin crosstalk has been established genetically and biochemically to regulate the progression of multiple diseases, including cancer ${ }^{10,11}$. While studies indicate the existence of EGFR-integrin interplay, the outcomes are attributed to signaling pathways away from the plasma membrane $7,12,13,14$. The impact of EGFR, or other growth factors, on cell mechanics remains largely unexplored in part due to the lack of tools to measure cellular forces and signaling outcomes. The challenge lies in identifying appropriate tools to study the communication between these parallel signaling paradigms and to quantify their specific contributions to cell mechanics.

Several approaches have been developed to measure forces generated by cell adhesion receptors, and the reader is directed to in-depth reviews of these techniques ${ }^{15,16}$. Briefly, traction force microscopy and micro-pillar array detection rely on the deformation of an underlying substrate to infer nanonewton $(\mathrm{nN})$ forces, an order of magnitude more than individual receptor forces ${ }^{17,18}$. Single-molecule techniques, including AFM and optical tweezers, are sensitive to single protein piconewton $(\mathrm{pN})$ forces but measure only one receptor at a time and do not offer good (or any) spatial resolution. DNA-based molecular tension probes and tension gauge tether (TGT) probes offer $\mathrm{pN}$ force resolution with diffractionlimited (or better) spatial resolution, giving them a unique role in studying single-cell forces ${ }^{19,20}$ from diverse cell types, including fibroblasts, cancer cells, platelets, and immune cells $^{21,22,23,24}$. While molecular tension probes have an extendable "spring" element, ideal for real-time imaging, TGT probes irreversibly rupture, leaving behind a fluorescent "force history". TGTs additionally modulate the tension threshold of the underlying substrate; a series of probes with similar chemical compositions but different rupture forces, or tension tolerances $\left(T_{\text {tol }}\right)$, can be used to quantify the minimum tension required for focal adhesion formation and cell spreading. TGT probes consist of two complementary DNA strands, one anchored to the surface and the other presenting a ligand to the cell. If a receptor binds the ligand and exerts a force greater than the $T_{\text {tol }}$ of the probe, the strands will be separated. $T_{\text {tol }}$ is defined as the constant force needed to rupture $50 \%$ of the probes in a $2 \mathrm{~s}$ interval under ideal conditions. In "turn-on" TGT probes, a quencher on the top strand can be separated from a fluorophore on the bottom strand. Only where the TGT probe has been ruptured, presumably by forces greater than or equal to $T_{\text {tol }}$, will a fluorescent signal be generated. TGT probes can also be fixed, allowing for easy manipulation of biological systems 
and testing of multiple conditions. For these reasons, TGT probes were used in this work.

TGT probes were employed to study how integrin-dependent cell adhesion and mechanical forces are modulated by activated EGFR ${ }^{21}$. This work established EGFR as a 'mechano-organizer', tuning focal adhesion organization and tension generation. Additionally, it was found that EGF stimulation influenced the distribution and maturity of focal adhesions and enhanced cell spreading. This approach could be used in future studies to investigate how growth factors influence mechanical forces in tumor progression and dynamics. While the role of EGFR-integrin crosstalk in regulating the epithelial to mesenchymal transition is established, the role of mechanical forces in this process remains under-explored ${ }^{10}$.

Here, a detailed protocol is presented for these experiments covering the synthesis and assembly of $56 \mathrm{pN}$ TGT probes, generation of TGT surfaces on glass coverslips, application of Cos-7 cells on the TGT surface and stimulation with EGF, fixation, and staining of cells with phalloidin, and an anti-paxillin antibody, high-resolution total internal reflection fluorescence (TIRF) and reflection interference contrast microscopy (RICM) imaging, and image quantification. This protocol, though written to investigate EGF stimulation of Cos-7 cells, is readily adaptable for many TGT based experiments. Different ligands, $T_{\text {tol }}$, cell types, stimulation parameters, proteins labeled following fixation, and quantitative analysis can be easily substituted in, making this protocol robust and widely useful.

\section{Protocol}

\section{TGT oligonucleotide preparation}

NOTE: The details of oligonucleotide probe synthesis are outlined here. Please note some modifications and purification steps can be outsourced for custom synthesis.

1. Activate the primary amine of the cyclo[Arg-Gly-Asp-DPhe-Lys(PEG-PEG)] peptide with the azide-NHS linker as described by Zhang et $\mathrm{al}^{22}$ by mixing in a $1: 1.5$ ratio (100:150 nmoles) in a final volume of $10 \mu \mathrm{L}$ dimethylformamide. Add $0.1 \mu \mathrm{L}$ of the organic base triethylamine and incubate for $12 \mathrm{~h}$ at $4^{\circ} \mathrm{C}$.

2. Purify the product by reverse phase HPLC using 0.1 M TEAA (solvent A) and 100\% acetonitrile (solvent B) with a flow rate of $1 \mathrm{~mL} / \mathrm{min}$ and the initial condition of $10 \%$ solvent B set at a gradient of $0.5 \% / \mathrm{min}$. Combine the eluted peaks (absorbance at $203 \mathrm{~nm}$ ) and verify by MALDI-TOF mass spectrometry. The product is CRGDfKazide.

3. To generate the TGT top strand, combine cRGDfK-azide and alkyne-21-BHQ2 oligonucleotide (TGT top strand: 5Hexynyl/GTGAAATACCGCACAGATGCG/3BHQ_2) in a 2:1 ratio ( $200 \mu \mathrm{M}: 100 \mu \mathrm{M})$ in $100 \mu \mathrm{L}$ of $1 \times$ Phosphate Buffered Saline (PBS) with $5 \mathrm{mM}$ sodium ascorbate and $0.1 \mu \mathrm{M}$ preformed $\mathrm{Cu}$-THPTA. Allow the reaction to proceed for a minimum of $4 \mathrm{~h}$ at room temperature (RT) or overnight at $4{ }^{\circ} \mathrm{C}$.

4. Process the mixture through the $\mathrm{P} 2$ desalting gel to remove excess dye, by-products, organic solvent, and unreacted reagents. Prepare the centrifuge column with $650 \mu \mathrm{L}$ of pre-hydrated $\mathrm{P} 2$ gel by spinning it down at $18,000 \times g$ for $1 \mathrm{~min}$. Discard the flow-through liquid and 
add the reaction mixture. Spin again at $18,000 \times g$ for $1 \mathrm{~min}$ and collect the flow-through. Bring the reaction mixture to a final volume of $300 \mu \mathrm{L}$ with ultrapure water. NOTE: Pre-hydrate the P2 gel with water for $6 \mathrm{~h}$.

5. Purify the desalted reaction mixture by reverse-phase HPLC. The organic solvents used for this purification include $0.1 \mathrm{M}$ TEAA in $\mathrm{H}_{2} \mathrm{O}$ (solvent $\mathrm{A}$ ) and $100 \% \mathrm{MeCN}$ (solvent $\mathrm{B}$, or the mobile phase).

1. Prior to injecting the mixture, equilibrate the column with an initial condition of $10 \%$ Solvent B with a gradient of $1 \% / \mathrm{min}$. Adjust the flow rate to $1 \mathrm{~mL} / \mathrm{min}$. Inject the reaction mixture into the HPLC loop with a $500 \mu \mathrm{L}$ injection needle.

2. Collect the product by visualizing the peak absorption at $260 \mathrm{~nm}$ for the DNA and $560 \mathrm{~nm}$ for the BHQ2 quencher. Dry the eluted product overnight in a vacuum centrifugal concentrator.

6. Employ nucleophilic substitution to couple TGT bottom strand to Cy3B-NHS ester as described in $\mathrm{Ma}$ et. $\mathrm{al}^{25}$. Mix $100 \mu \mathrm{M}$ of the 56 pN TGT bottom strand (5Biosg/TTTTTT/iUniAmM/ CGCATCTGTGCGGTATTTCACTTT) with $50 \mu \mathrm{g}$ of Cy3B-NHS ester pre-dissolved in $10 \mu \mathrm{L}$ of DMSO. Adjust the $\mathrm{pH}$ of this mixture to 9 with $0.1 \mathrm{M}$ sodium bicarbonate and bring the final volume to $100 \mu \mathrm{L}$ with $1 \mathrm{x}$ PBS. Incubate the reaction mixture overnight at RT.

7. Purify the mixture sequentially using $P 2$ gel filtration and reverse-phase HPLC to separate unreacted reagents, salts, and organic solvents (described in steps 1.4 and 1.5).

8. Estimate the concentration of the purified oligonucleotide-dye conjugates by recording their absorbance at $260 \mathrm{~nm}$ using a spectrophotometer.
9. Characterize the purified products by MALDI-TOF mass spectrometry. Dissolve excess 3-hydroxypicolinic acid into TA50 solvent $(50: 50 \mathrm{v} / \mathrm{v}$ acetonitrile and $0.1 \%$ TFA in $\mathrm{ddH}_{2} \mathrm{O}$ ) to prepare the fresh MALDI matrix. Estimated and measured molecular weights for the labeled products are: cRGDfK-1-BHQ2 - 8157.9 (calculated), 8160.1 (found); Cy3B labeled 56 pN TGT - 10272.7 (calculated), 10295.8 (found).

10. Dissolve the top and the bottom strands separately in nuclease-free water at a concentration between 30-50 $\mu \mathrm{M}$. Use DNase-free pipette tips to avoid stock contamination. Store at $4{ }^{\circ} \mathrm{C}$ for short-term applications or $-20{ }^{\circ} \mathrm{C}$ for long-term applications. The stability of oligonucleotides is not affected by repeated freeze-thaw cycles.

\section{Surface preparation}

\section{Day 1:}

1. Place $25 \mathrm{~mm}$ glass coverslips (up to 8) into a polytetrafluoroethylene rack. Place the rack in a 50 $\mathrm{mL}$ borosilicate beaker containing $40 \mathrm{~mL}$ of 200 proof ethanol. Cover the beaker with paraffin film to avoid water from entering and sonicate at an operating frequency of $35 \mathrm{kHz}$ for 10-15 min at RT (Figure 1A).

2. Fill a $50 \mathrm{~mL}$ beaker with $40 \mathrm{~mL}$ of Piranha solution freshly prepared by mixing sulfuric acid and hydrogen peroxide in a 3:1 ratio in a pyrex beaker. Stir with a glass pipette. Transfer the coverslip rack into the beaker and incubate for 30 min at RT in the fume hood to etch the coverslip surface (Figure 1B).

NOTE: Wear full PPE, including a lab coat, gloves, and goggles, and work in the chemical fume hood. 
Add hydrogen peroxide to the acid slowly to prevent overheating of the solution.

3. After etching, use tweezers to transfer the coverslip rack to a beaker with ultrapure water. Repeat this step six times at $15 \mathrm{~s}$ intervals to completely neutralize the acid (Figure 1C).

NOTE: Leave the Piranha solution in the chemical fume hood overnight before discarding it into the acid waste container.

4. Visually inspect the coverslips to ensure the surfaces look clean with no patterns or dust particles on the glass surface. Repeat steps 2.1-2.4 if patterns or dust are detected.

NOTE: Test surface hydrophilicity by dipping treated coverslips in water and removing them vertically. Water on treated coverslips recedes as a uniform sheet to form Young's rings compared to untreated coverslips that form patches.

5. Transfer the coverslip rack to a beaker with 200 proof ethanol and wash twice for $15 \mathrm{~s}$ to equilibrate surfaces to organic solvent. Transfer the coverslip rack into 200 proof ethanol solution with $3 \%$ APTES for $1 \mathrm{~h}$ at RT to silanize the coverslips (Figure 1D). Cover the beaker with paraffin film.

NOTE: APTES deposition parameters vary depending on the surface cleaning method, solvent water content, APTES concentration, incubation times, and temperature for annealing.

6. Immerse the rack in a clean beaker with 200 proof ethanol solution. Repeat this wash three times for $15 \mathrm{~s}$ each (Figure 1E).

7. Dry the coverslips using nitrogen $\left(\mathrm{N}_{2}\right)$ gas with low exit pressure. Place the coverslips into a $10 \mathrm{~cm}$ polystyrene dish with a piece of paraffin film laid flat inside it. Ensure the coverslips are dry and separated (Figure 1F).

8. Add $100 \mu \mathrm{L}$ of $2 \mathrm{mg} / \mathrm{mL}$ NHS-biotin solution in DMSO to four coverslips placed on paraffin film. Set a "sandwich" with the other four coverslips on top (two coverslips facing toward each other with the functionalization solution in between) and incubate the dish overnight at $4{ }^{\circ} \mathrm{C}$ (Figure 1G).

NOTE: At $4{ }^{\circ} \mathrm{C}$, the NHS reagent is more stable, which facilitates uniform surface functionalization. Additionally, the sandwich conserves reagents. Avoid adding excess solution in the sandwich as it may leak out and cause coverslips to slip.

\section{Day 2:}

9. Remove the dish from $4{ }^{\circ} \mathrm{C}$ and separate the sandwiched coverslips. Orient the slips in the rack with the coated surface facing each other as shown in Figure 2A. Wash them with 200 proof ethanol solution three times for $15 \mathrm{~s}$ each. Dry with $\mathrm{N}_{2}$ gas and place them in a new dish with a paraffin film inside it.

NOTE: Orienting the coverslips as indicated helps identify the functionalized surface.

10. Wash the coverslips with $1 \mathrm{~mL}$ of $1 \times$ PBS three times to equilibrate them back to the aqueous phase. Add $800 \mu \mathrm{L}$ of $0.1 \%$ bovine serum albumin (BSA) in $1 \times$ PBS $(w / v)$ to each of the coverslips and incubate at RT for $30 \mathrm{~min}$ to passivate the surface and block non-specific binding of subsequent functionalization reagents (Figure 2B).

11. After incubation, wash the coverslips three times with 1 $\mathrm{mL}$ of $1 \mathrm{x}$ PBS. Add $800 \mu \mathrm{L}$ of $1 \mu \mathrm{g} / \mathrm{mL}$ of streptavidin in $1 \mathrm{x}$ PBS at RT for 45-60 min to functionalize the coverslips (Figure 2C). 
NOTE: Retain one coverslip without streptavidin to verify passivation efficiency (optional). Add10 nM biotinylated molecules and image using experimental conditions. This surface intensity should be close to the camera's dark noise.

12. Simultaneously with step 2.11 , assemble the TGT probes (top: bottom strand at 1:1 molar ratio) at a final concentration of $50 \mathrm{nM}$ in $100 \mu \mathrm{L}$ of $1 \mathrm{M} \mathrm{NaCl}$ in a PCR tube using a thermocycler. Dissociate strands at $95^{\circ} \mathrm{C}$ for $5 \mathrm{~min}$, and gradually anneal by reducing the temperature to $25^{\circ} \mathrm{C}$ and maintaining it for $25 \mathrm{~min}$ (Figure 2D). Avoid extended exposure of TGT probes to light.

13. After streptavidin incubation, use $1 x$ PBS to wash the coverslips three times. Add $100 \mu \mathrm{L}$ of the preassembled TGT probes to four of the coverslips and make sandwiches using the remaining 4 coverslips with the functionalized side facing the probes (eight surfaces require four tubes of hybridized TGT probes). Cover with aluminum foil and incubate for $1 \mathrm{~h}$ at RT to allow probe binding to the surface (Figure 2E).

14. After incubation, separate the sandwiches and wash the coverslips with $1 \mathrm{x}$ PBS three times. The TGT surfaces are now ready for imaging. Carefully assemble the coverslips into precleaned imaging chambers and add $1 \mathrm{x}$ PBS to keep the surfaces hydrated (Figure 2F).

NOTE: Overtightening the chambers will crack the surface. Prevent drying of the surfaces.

\section{Cell preparation and staining}

1. To investigate the effect of the epidermal growth factor (EGF) stimulation on Cos-7 mechanics, adhesion, and cell spreading, trypsinize Cos-7 cells with $0.05 \%$ TrypsinEDTA for 2 min. Neutralize the trypsin by washing with
HBSS and centrifuging at $800 \times g$ for $5 \mathrm{~min}$. Repeat the neutralization step once more.

2. Plate cells at a density of $4 \times 10^{4}$ cells on the assembled TGT surfaces in Dulbecco's Modified Eagle Medium (DMEM) supplemented with $50 \mathrm{ng} / \mathrm{mL}$ EGF or DMEM without EGF. Allow the cells to spread for $60 \mathrm{~min}$ at 37 ${ }^{\circ} \mathrm{C}$ with $5 \% \mathrm{CO}_{2}$ in a cell culture incubator (Figure $3 \mathrm{~A}$ ). NOTE: Cells are incubated in DMEM without serum to avoid stimulation from growth factors. EGF is diluted in $10 \mathrm{mM}$ acetic acid to make a stock of $1 \mathrm{mg} / \mathrm{mL}$. It is used at $50 \mathrm{ng} / \mathrm{mL}$ in DMEM for imaging experiments.

3. Following incubation, wash the cells three times with $1 x$ PBS and fix with $2 \mathrm{~mL}$ of $4 \%$ paraformaldehyde for 12 min at RT (Figure 3B).

NOTE: All incubation steps are performed on a rotary shaker at $\sim 35 \mathrm{rpm}$ for uniform spreading of the solutions. Protect the TGT surfaces from light by covering them till they are ready for imaging.

4. Aspirate the fixative and wash the coverslips five times with $1 \times$ PBS at 5 min intervals at RT. Optionally, incubate the coverslips with $50 \mathrm{mM} \mathrm{NH}_{4} \mathrm{Cl}$ in $1 \mathrm{x}$ PBS for 30 min at $37{ }^{\circ} \mathrm{C}$ to quench paraformaldehyde-associated autofluorescence and wash three times with $1 \times$ PBS at 5 min intervals (Figure 3B).

5. Add Buffer A (1x PBS, $5 \%$ normal horse serum, $5 \%$ normal goat serum, 1\% BSA, $0.025 \%$ Triton $X-100$ ) and incubate for $30 \mathrm{~min}$ at $37^{\circ} \mathrm{C}$ to block and permeabilize the cells. Wash three times with $1 \times$ PBS at 5 min intervals (Figure 3B).

6. Place the imaging chambers with coverslips in a humidity container. Dilute the primary anti-paxillin antibody (focal adhesion marker) at 1:250 in blocking buffer (1x PBS, 5\% normal horse serum, $5 \%$ normal goat serum, $1 \%$ BSA, 
$0.005 \%$ Triton $x-100)$. Incubate with $200 \mu \mathrm{L}$ of primary antibody solution per coverslip for $2 \mathrm{~h}$ at $37^{\circ} \mathrm{C}$ (Figure 3B).

NOTE: Do not let the surfaces dry out.

7. Wash the coverslips five times with $1 \mathrm{x}$ PBS at $5 \mathrm{~min}$ intervals and return them to the humidity container. Label cells simultaneously with a mixture of dye conjugated goat anti-rabbit secondary antibody at 1:800 dilution and dye conjugated phalloidin (actin) at 1:400 dilution in 200 $\mu \mathrm{L}$ of blocking buffer per coverslip. Incubate at $37^{\circ} \mathrm{C}$ for 60 min (Figure 3B).

8. Wash the surfaces five times with $1 x$ PBS at 5 min intervals and store at $4{ }^{\circ} \mathrm{C}$ until ready for imaging (Figure 3B).

NOTE: Image samples within 3 days of surface preparation to avoid signal degradation.

\section{Image acquisition}

1. Use an oil immersion $60 x$ objective with a high numerical aperture (1.49) on an inverted microscope with 488, 561, and 647 TIRF excitation, RICM excitation, a perfect focus system, and a digital camera.

1. Add oil to the objective, clean the coverslip bottom of the sample chamber, and place the sample on the stage. Focus on a cell and engage the perfect focus.

2. Put the microscope into the RICM imaging mode with epifluorescence excitation and a GFP filter cube with the emission filter removed. Align the RICM by closing and centering the epi-illumination aperture diaphragm.

3. Put the microscope in TIRF mode with laser excitation and a quad-pass TIRF filter cube. Focus the $488 \mathrm{~nm}$ laser to a small spot on the ceiling of the room and increase the angle of incidence until past the critical angle while monitoring fluorescence on the camera in live mode. Observe a sharp reduction in background fluorescence and a single in-focus plane when the critical angle is surpassed.

NOTE: TIRF excites a thin region $(\sim 100 \mathrm{~nm})$ closest to the sample-coverslip interface highlighting open TGT probes and focal adhesions while eliminating out-offocus fluorescence from within the cell. If TIRF is not available, epi-fluorescence may be used; however, signal-to-noise ratios will be lower.

4. Identify cells for imaging using the "live" mode of the camera using RICM.

5. Acquire the RICM image and TIRF images of actin (640 $\mathrm{nm}$ ex), integrin tension (561 nm ex), and paxillin (488 $\mathrm{nm}$ ex). Obtain images sequentially using an exposure time of $200 \mathrm{~ms}$.

NOTE: The exposure time is dependent on many factors, including the objective, laser power, emission filters, and camera sensitivity. The signal should be at least $2 x$ greater than the background. The background is around $1000 \mathrm{AU}$ so the signal should be at least 2000-3000 AU.

6. Repeat $4.4-4.5$ for at least 30 cells. Change coverslips, focus, and repeat 4.4-4.5.

\section{Data analysis}

NOTE: Perform quantitative image analysis using the Fiji software and the analysis using statistics software.

1. Open the image set for one cell.

2. Create a mask of the cell area (RICM mask) by tracing the boundary of the cell in the RICM image using the ImageJ Freehand selection tool. Save the region of interest $(\mathrm{ROI})$ to the $\mathrm{ROI}$ manager (Analyze > Tools > ROI manager) (Figure 4A1,2). 
3. Pick a representative area outside the cell in the integrin tension image and draw a ROI of at least $200 \times 200$ pixels. Exclude any other cells or fluorescent debris from the ROI. Measure the background fluorescence in the ROI using the measure tool (Analyze > Measure) (Figure 4A3).

4. Subtract the mean background fluorescence obtained in step 5.2 from the tension image (Process > Math > Subtract) (Figure 4A4).

5. Use the RICM mask established in step 5.2 to define the tension signal within the cell footprint (ROI manager > Select $>$ Apply mask $>$ Edit $>$ Clear outside) (Figure 4A5).

6. Create a threshold mask for the tension image using Huang's method for auto thresholding (Image > Adjust $>$ Threshold) (Figure 4A6). Ensure that the threshold mask best represents the area for the generated integrin tension. As a rule of thumb, set the threshold to $2 x$ the mean background fluorescence.

7. Create a selection of the thresholded tension mask (Edit $>$ Selection $>$ Create) (Figure 4A7).

8. Transfer the selected mask onto the tension image generated in step 5.4 and measure the integrated intensity of open probes (ROI manager > Select $($ Tension Mask) $>$ Analyze $>$ Measure $>$ RawlntDen) (Figure 4C).

9. Measure the cell morphometric properties area, circularity, and aspect ratio from the RICM mask (ROI manager > Select $(\mathrm{RICM}$ mask) > Apply mask > Analyze $>$ Measure) (Figure 4B).

10. Measure the mechanical rupture density, defined as the percent of the cell footprint with ruptured probes by selecting the tension mask image and applying the RICM mask $($ ROI manager $>$ Select $(\mathrm{RICM}$ Mask $)>$ Analyze $>$ Measure $>$ \%Area) (Figure 4C).

11. Export the measurements for further analysis and visualization into statistics software.

12. Repeat 5.1-5.11 for every cell.

\section{Representative Results}

Turn-on TGT probes were used to investigate the effect of ligand-activated epidermal growth factor receptor (EGFR) on integrin-mediated cell mechanics and adhesion formation in Cos-7 cells ${ }^{21}$. The probes present the ligand cyclic ArgGly-Asp-Phe-Lys (cRGDfK) $21,23,25,26$, which is selective for the integrin heterodimer $\alpha \mathrm{V} \beta 3$ with only a small affinity for $\alpha 5 \beta 1$ integrins $27,28,29,30$. The TGT probe comprises a duplex DNA functionalized onto a glass coverslip surface via the bottom strand using biotin-streptavidin binding. The top strand displays the integrin ligand and is available to bind to the cognate integrin receptor on the cell membrane (Figure 5A). The bottom strand is labeled with a fluorophore and the top strand with a quencher, leading to minimal background fluorescence when the duplex TGT is intact. If an integrin binds the ligand and applies a force with a magnitude larger than the $T_{\text {tol }}$ of the probe, the DNA duplex will separate leading to fluorescence (Figure 5A). Any TGT probe that has not been ruptured by a mechanical force will remain nonfluorescent. This force selective turn-on fluorescence allows systematic and quantitative mapping of $\mathrm{pN}$ scale integringenerated forces at diffraction-limited resolution. TGT probes additionally modulate the tension threshold of the substrate.

Shown here is a representative example of a TGT surface with a $\mathrm{T}_{\text {tol }}$ of $56 \mathrm{pN}$. Cos-7 cells were plated on this TGT surface with or without EGF stimulation to study the 
impact of EGFR activation with ligand stimulation on cell adhesion and integrin mechanics (Figure 5A,B). The cells were incubated with or without EGF on the TGT surfaces for 60 mins, fixed, and immuno-stained to display the focal adhesion distribution (paxillin) and the organization of the cytoskeleton (F-actin) (Figure 5B). The cells were then imaged using RICM and TIRF microscopy. As clearly seen in the RICM image, Cos-7 cell spreading on the $56 \mathrm{pN}$ TGT surface was significantly enhanced with EGF stimulation compared to without stimulation. This was quantified for 50 cells in each condition by measuring the size of the cellsubstrate contact region from the RICM image (Figure 5C). Stimulation with EGF resulted in a more circular morphology, representative of Cos-7 cells spreading and growing in their natural physiological environment (Figure 5D). The fluorescence from open probes is also higher with EGF stimulation as observed in the tension fluorescence image. The integrated intensity of open probes, which is proportional to the number of open probes, was much higher with EGF stimulation compared to without (Figure 5B,E). This is a representation of all the receptor-ligand engagements where integrins applied a force greater than $\mathrm{T}_{\text {tol }}(56 \mathrm{pN})$.

Staining with paxillin showed that the distribution, number, maturation (size), and organization of focal adhesions were also impacted by EGF stimulation. Focal adhesions in EGF stimulated cells appeared more mature and radially oriented compared to no EGF controls. The F-actin cytoskeletal organization was also enhanced with EGF stimulation, as assessed by the phalloidin staining (Figure 5B). These qualitative assessments were made by visual comparison of images from both treatment groups. Quantitative analysis of focal adhesion can be done but is beyond the scope of this protocol. In this experiment, the TGT surface provided a platform to systematically detail the effect of EGFR activation on cell spreading, integrin mechanics, and focal adhesion formation.

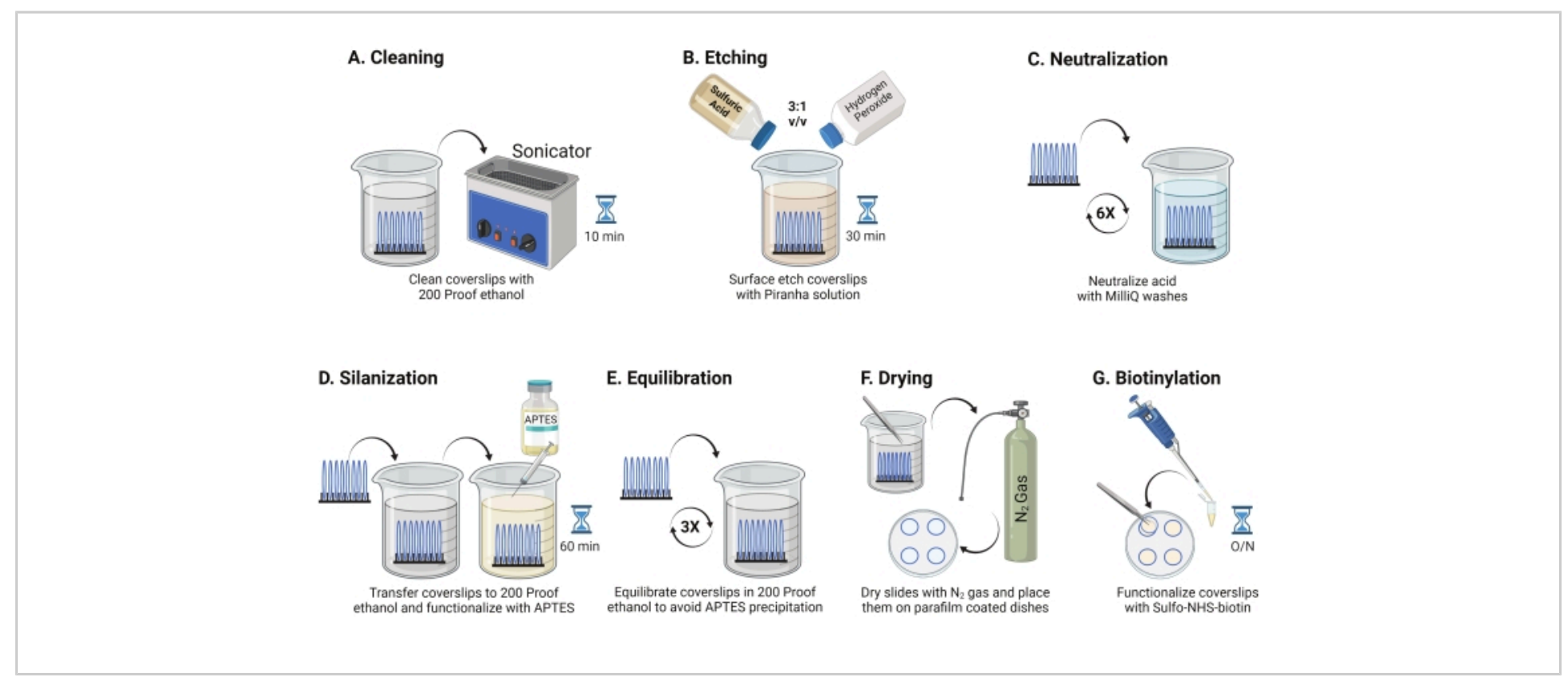


Figure 1: Schematic for Day 1 of the TGT surface preparation. (A) Clean the coverslips. (B) Etch the coverslip surface. (C) Neutralize the Piranha solution. (D) Silanize the surface to make reactive amine groups. (E) Equilibrate the coverslips to the organic phase. (F) Dry the coverslips with an inert gas. (G) Biotinylate the surface amine groups. Please click here to view a larger version of this figure.

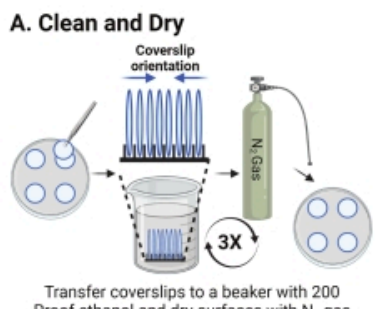
Proof ethanol and dry surfaces with $\mathrm{N}_{2}$ gas

D. Probe Assembly

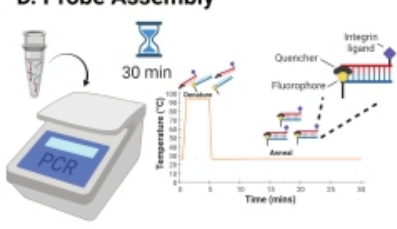

Assembly DNA based TGT probes in a thermocycler
B. Passivation

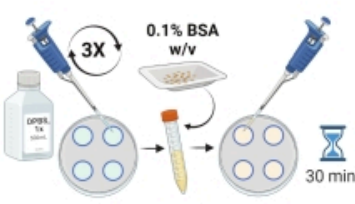

Wash coverslips with 1 X PBS and passivate surfaces with BSA

E. Probe binding on surface

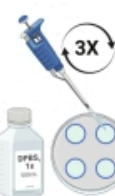

Functionalize coverslips with assembled tension gauge tether probes
C. Functionalization with streptavidin

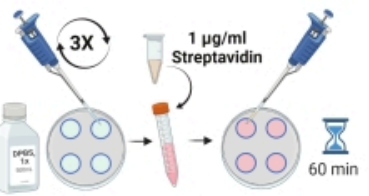

Wash coverslips with 1 X PBS and Wash coverslips with $1 \times$ PBS and
functionalize coverslips with streptavidin

F. Assemble imaging chamber

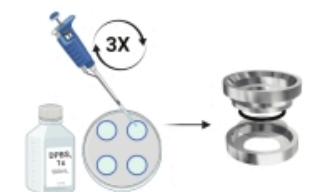

Wash coverslips with $1 X$ PBS and assemble the TGT surface in atto fluor chamber

Figure 2: Schematic for Day 2 of the TGT surface preparation. (A) Clean and dry the coverslips to remove any residual biotin from the day before. (B) Passivate with BSA to prevent non-specific binding of reagent in subsequent steps. (C) Functionalize the coverslips with streptavidin. (D) Hybridize the probes in a thermo cycler. (E) Apply the synthesized probes to the coverslips (F) Assemble the coverslip in the cell imaging chamber. Please click here to view a larger version of this figure. 

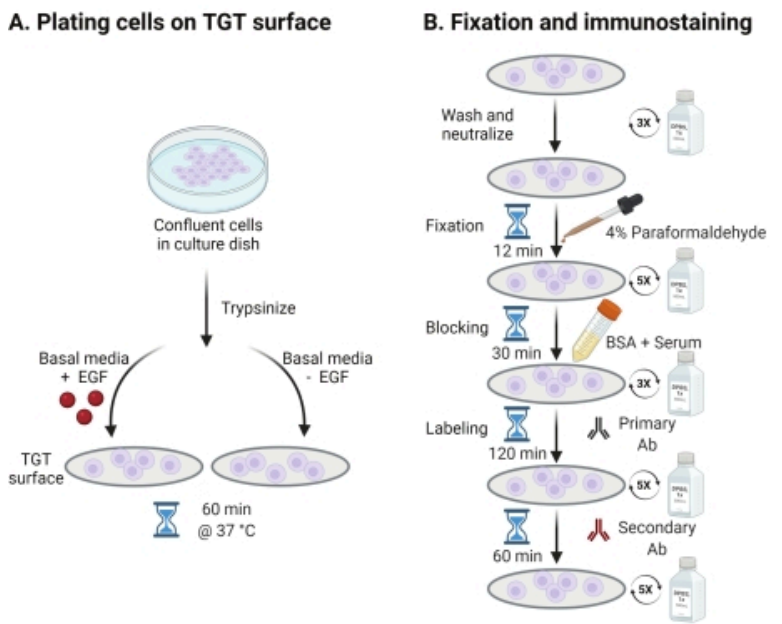

C. Imaging

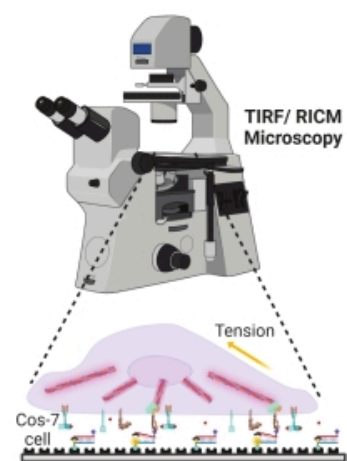

Figure 3: General workflow highlighting the broad steps across the entire experimental setup. (A) Process for cell detachment and plating on the TGT surface in basal media (DMEM) with or without EGF stimulation. (B) Flowchart of the steps involved in fixation and immuno-staining post-attachment and spreading on the TGT surface. (C) Post-staining, cells are imaged on an inverted fluorescence microscope with RICM and TIRF microscopy. Please click here to view a larger version of this figure. 


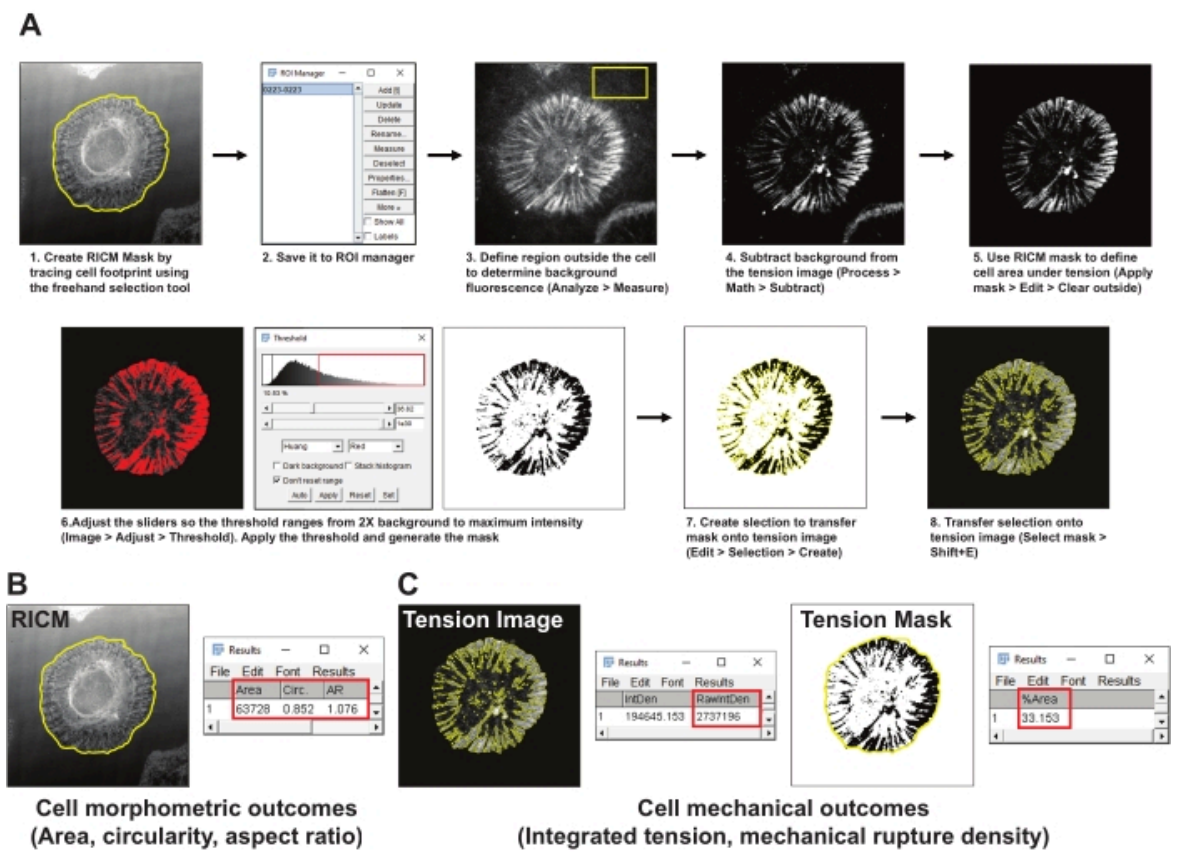

Figure 4: Example of data processing and quantitative analysis. (A) Step-by-step breakdown of the analysis pipeline employed in Fiji (ImageJ) for RICM and tension image quantification. (B) A representative example for cell morphometric outcomes analyzed using the above pipeline. (C) Representative examples for cell mechanical outcomes analyzed using the above-mentioned pipeline. Please click here to view a larger version of this figure. 

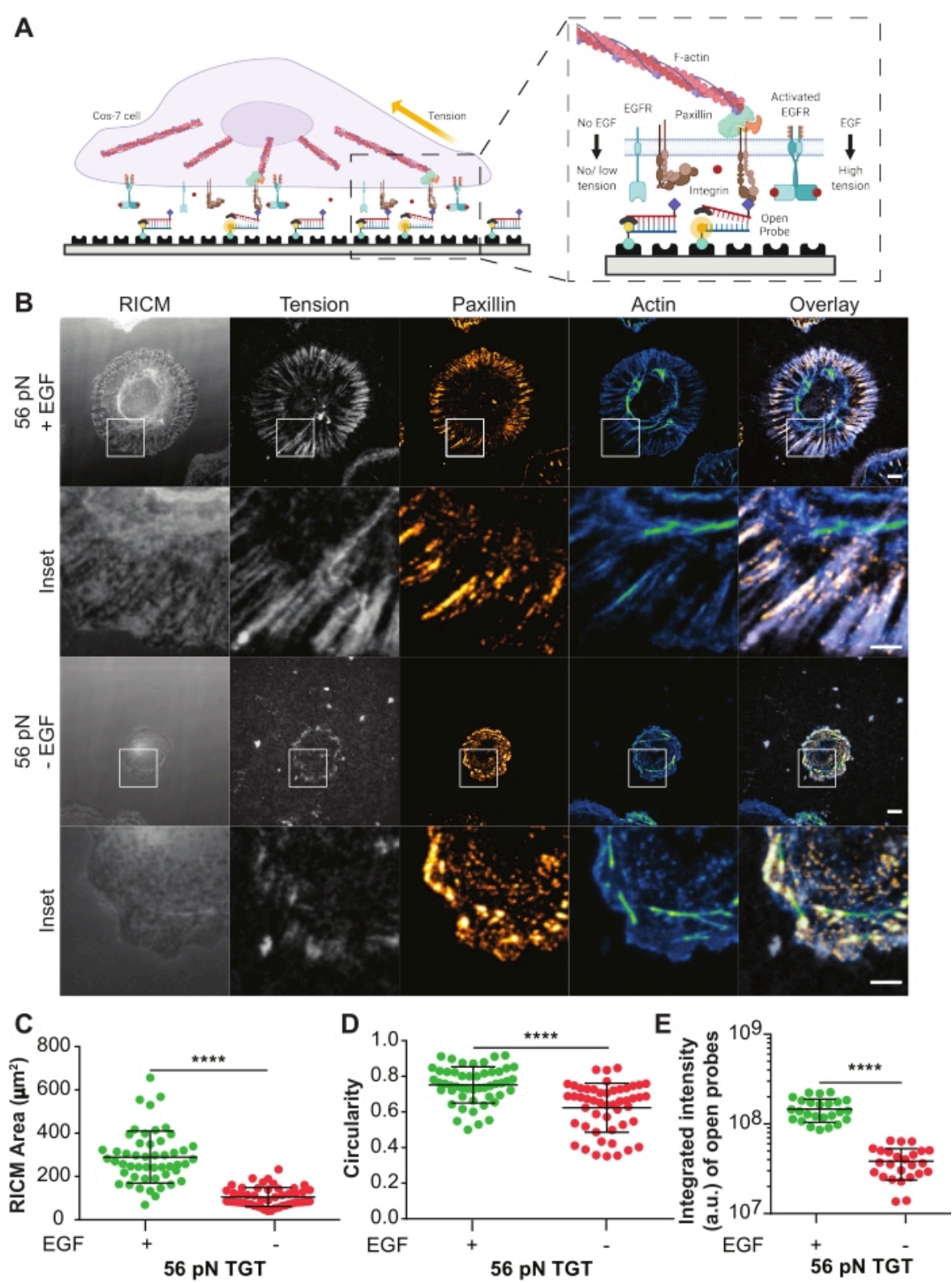

Figure 5: Example data from a TGT experiment. (A) Diagram highlighting the contact zone at the cell membraneTGT surface interface. Inset projects integrins interacting with its cognate ligand cRGDfK with (right) or without (left) EGF stimulation. (B) RICM and TIRF images of Cos-7 cells spread on the $56 \mathrm{pN}$ TGT surface. The images are obtained 60 min post-plating with or without EGF stimulation. Individual RICM (as acquired), integrin tension (grayscale), paxillin (orange hot), and F-actin (blue-green) images are shown with overlays for both stimulation conditions. Scale Bar: $10 \mu \mathrm{m}$. The inset highlights a zoomed-in ROI (region of interest) detailing the colocalization of the generated integrin tension at sites of adhesion formation marked by paxillin, and the underlying subcellular cytoskeletal organization marked by actin. Scale Bar: 5 $\mu \mathrm{m}$. (C-E) Scatter plots for the spread area (RICM cell footprint) (C), circularity (D), and integrated tension (E) for Cos-7 cells 
with or without EGF stimulation. Bars indicate mean \pm s.d. Differences between the groups were assessed statistically with Student's $t$-test; ${ }^{* \star * *} \mathrm{P}<0.0001 . \mathrm{n}=50$ cells across three independent experiments. Please click here to view a larger version of this figure.

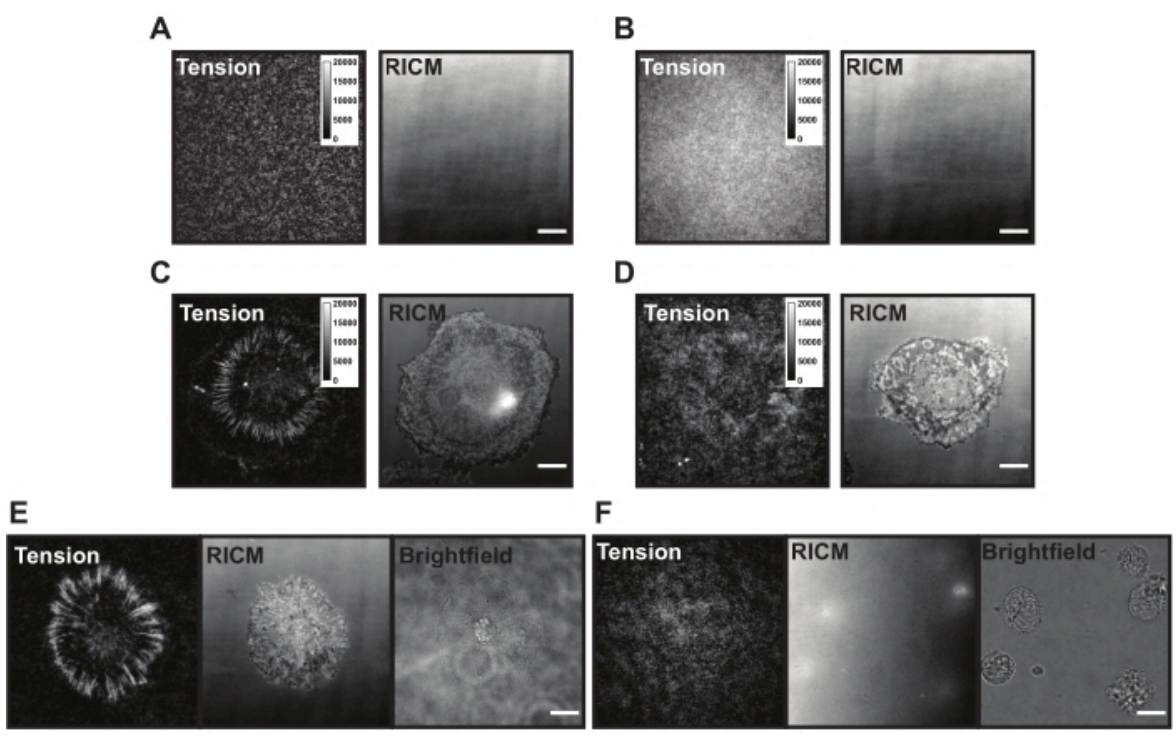

Figure 6: Example TGT surfaces with different possible problems. (A) Tension and RICM images of an ideal TGT surface with assembled probe quenched before cell adhesion. (B) Tension and RICM images of a TGT surface where the TGT probe lacks the top strand (quencher). Tension image shows uniform fluorescence from open fluorophore in the bottom strand. (C) Tension and RICM images for cells spread on an ideal TGT surface. (D) Tension and RICM images for cells spread on a poorly made TGT surface with limited passivation or degraded probe. (E) Tension, RICM, and brightfield images for cells plated on an ideal surface with cRGDfK ligand indicating cRGDfK-integrin interactions are vital for cell attachment and tension generation. (F) Tension, RICM, and brightfield images for cells plated on a surface without cRGDfK ligand on the TGT. While the cells are visible in the brightfield image, no cell attachment or generated integrin tension is observed. Scale Bar: $10 \mu \mathrm{m}$. Please click here to view a larger version of this figure.

\section{Discussion}

With the detailed step-by-step procedure outlined above, one can prepare TGT surfaces to quantify cell morphology and integrin tension generated by adherent cells during cell attachment and spreading following treatment with EGF. The straightforward probe design and synthesis and surface preparation along with the simple experimental setup provided a stable platform to study the interaction of EGFR and integrins. Overall, the results validate that liganddependent activation of EGFR enhances cell spreading, tunes the force-bearing properties of integrin receptors, and promotes focal adhesion organization and maturation. The results obtained using TGT probes support the overarching hypothesis that growth factors, such as EGFR, act as 'mechano-organizers', increasing the amount and spatial 
organization of integrin tension and regulating the orientation and mechanics of focal adhesions.

Upon application onto the TGT surface, the cells land, attach, and spread as the integrin $(\alpha \vee \beta 3)$ receptors sense and bind to the cRGDfK ligand. In doing so the TGT probes can be mechanically ruptured, generating fluorescence at the site of ligand engagement. The readout is the cumulative "force history" of the cell interacting with the surface. There are some common issues with the TGT surfaces that can be present during these experiments. High surface background fluorescence (Figure 6A,B), patchy surface appearance, failure of the cells to generate tension signal (Figure 6C,D), and failure of cells to spread (Figure 6E,F) may be due to technical shortcomings with the TGT probe or surface synthesis. Solutions to these common issues are presented in Table 1.

The straightforward design of TGT probes provides cell biologists with a powerful tool to study specific growth factorintegrin signaling outcomes in isolation without interference from other cell surface receptors by providing only specific ligands and stimulations. Additionally, TGT probes allow investigation of the tension threshold underlining individual integrin receptors during cell adhesion at $\mathrm{pN}$ sensitivity. Alternate approaches fail to report forces exerted by individual receptors with high spatial resolution in fixed samples ${ }^{31}$. Traction force microscopy is only sensitive to $\mathrm{nN}$ forces, an order of magnitude higher than the forces applied by individual integrin receptors ${ }^{15}$, and molecular tension probes measure $\mathrm{pN}$ forces, but because they are reversible, they do not robustly withstand fixation. For these reasons, TGT probes are an attractive tool to study the mechanics of growth factor-integrin interactions.
There are several technical nuances associated with TGT probes that should be considered before designing an experiment. The tension image is a snapshot in time, representing the force history and not an indicator of the receptor-ligand engagements at any given time point. Since signal generation is dependent on probe separation, the TGT fluorescence results from open probes not under active tension from receptor-ligand engagement. This means the readout for integrin tension obtained on the TGT surface is historical and cumulative in nature representing where there were forces larger than $T_{\text {tol}}$; the locations of current receptor-ligand forces less than $T_{\text {tol }}$ are not reported ${ }^{19,32}$. Because TGT rupture results in termination of the receptorligand engagement, cell spreading is due to integrin-ligand interactions that experience forces lower than $T_{t o l}$. The user must therefore be careful when defining the time postplating to estimate the mechanical outcomes associated with integrin-based adhesions. Finally, the meaning of $T_{\text {tol }}$ must be considered. The TGT probes employed here have a $T_{\text {tol }}$ of $56 \mathrm{pN}$, where $\mathrm{T}_{\mathrm{tol}}$ is the constant force needed to rupture $50 \%$ of the probes when applied for $2 \mathrm{~s}$. When considering complicated biological systems, TGTs likely experience a heterogeneous and diverse force gradation with varying time dependencies. If TGTs are ruptured by forces larger than $T_{\text {tol }}$, the fluorescence would be an underestimation of the total tension. Alternatively, forces below $\mathrm{T}_{\text {tol }}$ applied for longer durations can rupture a similar number of probes as high threshold forces applied for shorter times. Both these scenarios may result in the same fluorescence intensity readout, making it difficult to resolve the exact tension magnitude or dynamics using TGT probes ${ }^{33,34}$.

Overall, assessments of integrin tension with growth factor stimulation should be made carefully by designing experiments with internal controls, comparing spreading 
profiles on other matrix-coated surfaces, making parallel assessments of TGT fluorescence in cells in the presence or absence of growth factor stimulation, and using TGTs with different $T_{\text {tol. }}$ TGTs allow quantification of the role of growth factor signaling in regulating the mechanics of integrin receptors, focal adhesion dynamics, and cell spreading. This protocol can be used as a template for many TGT-based experiments using probes with different $\mathrm{T}_{\text {tol }}$, different ligands, different cell types, or different stimulation conditions. Any proteins of interest can be labeled following fixation, and any type of quantitative image analysis may be implemented. As such, we present a template for numerous TGT experiments.

The use of TGT probes is not limited to studying integrins but can be extended to a diverse array of cell membrane receptors across different cell types by modifying the ligand. TGT probes have been used to investigate the role of forces in regulating various receptor signaling cascades, including identifying the mechanical role of Notch receptor mechanics in embryonic development and neurogenesis ${ }^{35}$, the forces mediating identification and internalization of antigens by $B$ cell receptors $^{36}$, and the mechanical proof-reading ability of T-cell surface receptors to detect changes in forces to boost the strength and specificity of signal transfer ${ }^{37}$. Together, these findings highlight the immense potential of TGT probes in a variety of experimental settings.

\section{Disclosures}

The authors declare no conflicts of interest.

\section{Acknowledgments}

The authors would like to recognize the members of the Mattheyses laboratory for fruitful discussions and critiques.
We acknowledge funding to A.L.M. from NSF CAREER 1832100 and NIH R01GM131099.

\section{References}

1. Lim, C.-G., Jang, J., Kim, C. Cellular machinery for sensing mechanical force. BMB Reports. 51 (12), 623-629 (2018).

2. Moraes, C., Sun, Y., Simmons, C. A. (Micro)managing the mechanical microenvironment. Integrative Biology. 3 (10), 959-971 (2011).

3. Vogel, V., Sheetz, M. P. Mechanical forces matter in health and disease. From Cancer to Tissue Engineering. In Nanotechnology. 233-303 (2010).

4. Wang, J. H. C., Li, B. Mechanics rules cell biology. BMC Sports Science, Medicine and Rehabilitation. 2 (1), 16 (2010).

5. Moeendarbary, E., Harris, A. R. Cell mechanics: principles, practices, and prospects. Wiley Interdisciplinary Reviews: Systems Biology and Medicine. 6 (5), 371-388 (2014).

6. Streuli, C. H., Akhtar, N. Signal co-operation between integrins and other receptor systems. Biochemical Journal. 418 (3), 491-506 (2009).

7. Chiasson-MacKenzie, C., McClatchey, A. I. EGFRinduced cytoskeletal changes drive complex cell behaviors: The tip of the iceberg. Science Signaling. 11 (515), eaas9473 (2018).

8. Kechagia, J. Z., Ivaska, J., Roca-Cusachs, P. Integrins as biomechanical sensors of the microenvironment. Nature Reviews Molecular Cell Biology. 20 (8), 457-473 (2019). 
9. De Luca, A. et al. The role of the EGFR signaling in tumor microenvironment. Journal of Cellular Physiology. 214 (3), 559-567 (2008).

10. Javadi, S., Zhiani, M., Mousavi, M. A., Fathi, M. Crosstalk between Epidermal Growth Factor Receptors (EGFR) and integrins in resistance to EGFR tyrosine kinase inhibitors (TKIs) in solid tumors. European Journal of Cell Biology. 99 (4), 151083 (2020).

11. Eliceiri, B. P. Integrin and growth factor receptor crosstalk. Circulation Research. 89 (12), 1104-1110 (2001).

12. Dan, L., Jian, D., Na, L., Xiaozhong, W. Crosstalk between EGFR and integrin affects invasion and proliferation of gastric cancer cell line, SGC7901. OncoTargets and Therapy. 5, 271-277 (2012).

13. Giancotti, F. G., Tarone, G. Positional control of cell fate through joint integrin/receptor protein kinase signaling. Annual Reviews: Cell and Developmental Biology. 19, 173-206 (2003).

14. Ricono, J. M. et al. Specific cross-talk between epidermal growth factor receptor and integrin alphavbeta5 promotes carcinoma cell invasion and metastasis. Cancer Research. 69 (4), 1383-1391 (2009).

15. Polacheck, W. J., Chen, C. S. Measuring cell-generated forces: a guide to the available tools. Nature Methods. 13 (5), 415-423 (2016).

16. Hang, $X$. et al. Nanosensors for single cell mechanical interrogation. Biosensors and Bioelectronics. 179, 113086 (2021).

17. Style, R. W. et al. Traction force microscopy in physics and biology. Soft Matter. 10 (23), 4047-4055 (2014).
18. Schoen, I., Hu, W., Klotzsch, E., Vogel, V. Probing cellular traction forces by micropillar arrays: contribution of substrate warping to pillar deflection. Nano Letters. 10 (5), 1823-1830 (2010).

19. Ma, V. P.-Y., Salaita, K. DNA Nanotechnology as an Emerging Tool to Study Mechanotransduction in Living Systems. Small. 15 (26), 1900961 (2019).

20. Kim, Y., Kim, K. A., Kim, B. C. Double-stranded DNA force sensors to study the molecular level forces required to activate signaling pathways. Journal of the Korean Physical Society. 78 (5), 386-392 (2021).

21. Rao, T. C. et al. EGFR activation attenuates the mechanical threshold for integrin tension and focal adhesion formation. Journal of Cell Sciences. 133 (13), jcs238840 (2020).

22. Zhang, Y., Ge, C., Zhu, C., Salaita, K. DNA-based digital tension probes reveal integrin forces during early cell adhesion. Nature Communications. 5, 5167 (2014).

23. Liu, Y. et al. DNA-based nanoparticle tension sensors reveal that $\mathrm{T}$-cell receptors transmit defined $\mathrm{pN}$ forces to their antigens for enhanced fidelity. Proceedings of the National Academy of Sciences of the United States of America. 113 (20), 5610-5615 (2016).

24. Zhang, Y. et al. Platelet integrins exhibit anisotropic mechanosensing and harness piconewton forces to mediate platelet aggregation. Proceedings of the National Academy of Sciences of the United States of America. 115 (2), 325-330 (2018).

25. Ma, V. P.-Y. et al. Mechanically induced catalytic amplification reaction for readout of receptor-mediated cellular forces. Angewandte Chemie International Edition. 55 (18), 5488-5492 (2016). 
26. Wang, X., Ha, T. Defining single molecular forces required to activate integrin and notch signaling. Science. 340 (6135), 991-994 (2013).

27. Chen, Y., Lee, H., Tong, H., Schwartz, M., Zhu, C. Force regulated conformational change of integrin $\alpha \mathrm{V} \beta 3$. Matrix Biology. 60 - 61, 70-85 (2017).

28. Kantlehner, M. et al. Surface coating with cyclic RGD peptides stimulates osteoblast adhesion and proliferation as well as bone formation. ChemBioChem. 1 (2), 107-114 (2000).

29. Kapp, T. G. et al. A comprehensive evaluation of the activity and selectivity profile of ligands for RGD-binding integrins. Scientific Reports. 7, 39805 (2017).

30. Kok, R. J. et al. Preparation and functional evaluation of RGD-modified proteins as alpha(v)beta(3) integrin directed therapeutics. Bioconjugate Chemistry. 13 (1), 128-135 (2002).

31. Li, I. T. S., Ha, T., Chemla, Y. R. Mapping cell surface adhesion by rotation tracking and adhesion footprinting. Scientific Reports. 7 (1), 44502 (2017).

32. Wang, Y. et al. Force-activatable biosensor enables single platelet force mapping directly by fluorescence imaging. Biosensors and Bioelectronics. 100, 192-200 (2018).

33. Murad, Y., Li, I. T. S. Quantifying molecular forces with serially connected force sensors. Biophysical Journal. 116 (7), 1282-1291 (2019).

34. Yasunaga, A., Murad, Y., Li, I. T. S. Quantifying molecular tension-classifications, interpretations and limitations of force sensors. Physical Biology. 17 (1), 011001 (2019).
35. Luca, V. C. et al. Notch-Jagged complex structure implicates a catch bond in tuning ligand sensitivity. Science. 355 (6331), 1320-1324 (2017).

36. Spillane, K. M., Tolar, P. B cell antigen extraction is regulated by physical properties of antigen-presenting cells. The Journal of Cell Biology. 216 (1), 217-230 (2017).

37. Brockman, J. M., Salaita, K. Mechanical proofreading: a general mechanism to enhance the fidelity of information transfer between cells. Frontiers in Physics. 7, 14 (2019). 